\title{
Propriété intellectuelle et sélection dans les pays du Sud
}

\section{Plant property rights and breeding in developing countries}

Oléagineux, Corps Gras, Lipides. Volume 8, Numéro 5, 546-50, Septembre - Octobre 2001, Dossier : Aspects des filières semencières Nord/Sud

Auteur(s) : Henri FEYT, Cirad-Amis, Programme Biotrop, avenue Agropolis TA 40/03, 34398 Montpellier Cedex 5, France.

Résumé : Avec les progrès de la science et des technologies, nombre de produits marchands qui étaient considérés jusqu'ici comme peu élaborés, incorporent aujourd'hui dans leur valeur une part sans cesse croissante due à l'ingéniosité humaine et aux inventions : c'est en particulier le cas des variétés végétales. Par ailleurs, le formidable développement des échanges d'idées et de marchandises ainsi que de la circulation des personnes, que nous connaissons sous le terme de mondialisation, a posé le problème de la protection de la propriété intellectuelle au niveau mondial. De ce constat est né l'accord ADPIC1, préparé durant le cycle 1986-1994 de négociation de I'OMC, connu sous le nom de "Uruguay round ", signé à Marrakech en 1994 et entré en vigueur le 1er janvier 1995. Cet accord fait obligation à tous ses membres signataires de mettre en place un système global de protection de la propriété intellectuelle. Dans le cas particulier des variétés végétales, il prévoit : Article 27.3. «[...] Les Membres pourront aussi exclure de la brevetabilité : [...] b) Les végétaux et les animaux autres que les micro-organismes, et les procédés essentiellement biologiques d'obtention de végétaux ou d'animaux, autres que les procédés non biologiques et microbiologiques. Toutefois, les membres prévoiront la protection des variétés végétales par brevets, par un système sui generis efficace, ou par une combinaison de ces deux moyens. Les dispositions du présent alinéa seront réexaminées quatre ans après la date d'entrée en vigueur de I'Accord sur l'OMC. » Applicable un an après son entrée en vigueur, l'accord prévoyait pour les pays en développement une période supplémentaire de quatre ans (soit jusqu'au 1er janvier 2000), voire dix ans pour les pays les moins avancés, pour la mise en place d'un système de protection, ainsi qu'un réexamen de ces dispositions à l'aube du xxie siècle. Force est de constater que, si la révision de la partie de cet accord qui devait intervenir à partir de 1999 a bien été engagée, on est encore très loin de l'entrée en vigueur d'un système de protection des variétés végétales dans la plupart des pays du Sud. Cependant, les démarches sont très avancées pour nombre d'entre eux2, soutenues par des associations régionales ou sous-régionales de semenciers telles que I'APSA (Asia and Pacific Seed Association) ou l'AFSTA (African Seed Trade Association). Le mouvement est donc lancé et il est intéressant de se poser les questions suivantes à propos des pays dont les économies sont les moins avancées. La mise en place de ces droits est-elle prioritaire ? Quel en sera l'impact ? Suffira-t-elle à redynamiser la production agricole de ces pays ou faut-il l'accompagner par des dispositions complémentaires ? Comment la mettre en œuvre ? Nous essaierons de proposer quelques éléments de réponse à ces différentes questions. 
Summary : Do developing countries need plant variety protection rights? As a result of the Uruguay Round adopted in Marrakech in 1994, all WTO member countries should have implemented property rights systems before the year 2000. In this frame, countries are required to provide "for the protection of plant varieties by patents or by an effective sui generis system or by any combination thereof". The interest of implementing IPR on plant varieties in developing countries is discussed, considering two types of seedlings or seeds: those with a very high added value (vegetable, ornamental, industrial... crops) and those for stapple food crops with a very low added value. If IPR are needed at short notice in the first case, in the second case the priority is to set up a bottom-up seed production organization aiming to promote individual initiatives at the farmer's level with an adapted seed quality control system implemented by governments.

Keywords : seed, seed production organization and system, seed quality control, developing countries, plant variety protection rights.

\section{ARTICLE}

\section{Extrême diversité des variétés végétales}

Rappelons tout d'abord qu'il est impossible dans la pratique de parler de variété végétale sans parler de semences ou de plants. Pour prendre une comparaison avec l'informatique, on peut dire que la variété est un "programme génétique ", correspondant à ce que l'on appelait autrefois le software, et que la semence ou le plant est le support physique de ce programme, l'équivalent de la disquette, qui permet de multiplier et diffuser la variété. C'est pourquoi, dans la suite du texte, nous utiliserons le plus souvent le mot " semences " pour désigner l'ensemble semence/plant + variété, c'est-à-dire le programme génétique et son support physique.

Cela dit, la notion de variété recouvre un ensemble non pas homogène mais, au contraire, extrêmement divers, fonction de l'espèce végétale, de son mode de reproduction (autogamie, allogamie, reproduction végétative), du type variétal (lignée, population, hybride, clone, etc.). Pour le sujet qui nous intéresse ici, "protection des variétés et sélection dans les pays du Sud ", nous en ferons deux grandes catégories : d'une part, les variétés d'espèces vivrières, qui correspondent à des volumes de semences considérables et à faible valeur ajoutée par rapport au produit consommation car, en général, les semences et le produit consommé sont de même nature (grain, racine ou tubercule) ; d'autre part, les semences dites " fines " ou " techniques " (graines potagères et florales), les boutures et plants pour les espèces fruitières, les plantes ornementales ou les cultures industrielles, etc. qui se distribuent en général à l'unité et intègrent souvent une très forte valeur ajoutée.

Cette distinction est nécessaire car la problématique de la protection de la propriété intellectuelle des variétés végétales se présente en des termes complètement différents selon que l'on s'intéresse à l'une ou l'autre catégorie de ces semences. 


\section{Protection des variétés de semences à forte valeur ajoutée}

De multiples raisons militent en faveur de l'adoption par les pays du Sud d'un système de protection de la propriété intellectuelle des obtentions végétales.

Tout d'abord, ne serait-ce que pour leurs besoins propres, ces pays doivent impérativement avoir accès à ces semences à forte valeur ajoutée que leurs agriculteurs sont à même de parfaitement bien valoriser. De plus, la mise en œuvre de ces produits implique le plus souvent des techniques culturales innovantes et constituent par là même des vecteurs efficaces de développement.

Par ailleurs, sous peine de perdre leurs marchés à l'exportation, les pays du Sud sont obligés d'entrer dans le jeu de la concurrence, entre eux aussi bien qu'avec les pays du Nord, et donc de s'adapter à la demande des marchés. Ceux-ci deviennent de plus en plus exigeants, en termes de présentation ou de caractéristiques technologiques du produit, de prix, d'approvisionnement régulier et garanti, etc. Or, nombre de ces éléments sont fortement influencés, voire même commandés, par le choix variétal. Dans ce contexte, il convient de rappeler que l'Upov 1991 ainsi que les lois semencières de nombreux pays n'ayant pas encore adhéré à la version la plus récente de cette convention prévoient que le droit de l'obtenteur peut s'exercer, si ce dernier n'a pas pu le faire valoir plus tôt, sur le produit de la récolte et, à la discrétion de chaque État, sur le produit issu d'une première transformation de la récolte. Ainsi, une production qui serait obtenue dans un pays ne reconnaissant pas les droits de l'obtenteur, et en violation de ces droits, ne peut pas légalement être importée dans un autre pays disposant d'un système de protection.

On peut aussi facilement concevoir qu'un obtenteur, particulièrement de variétés de plantes autogames ou à reproduction végétative, ne sera guère enclin à diffuser ses variétés dans un pays ne respectant pas ses droits. Qui plus est, si ces pays correspondent à des types agronomiques ou technologiques plus ou moins spécifiques, compte tenu de leurs contraintes agro-climatiques, il ne sera pas porté à investir dans des programmes de sélection propres à ces zones. Finalement, cela se traduira par une baisse de la compétitivité des productions agricoles et une perte d'activité économique pour ces pays ${ }^{3}$.

Mais les pays du Sud sont déjà ou peuvent être aussi producteurs de semences. Nombre d'entre eux disposent en effet d'avantages comparatifs substantiels par rapport aux pays du Nord pour la production de semences très techniques : climat (ne serait-ce qu'en contre-saison), maind'œuvre disponible, coûts de production, etc. Là encore, les obtenteurs seront d'autant plus tentés de développer des activités de production dans ces conditions que ces pays auront adhéré à un système qui protège leurs droits.

Pour cet ensemble de raisons, les pays du Sud, en tant que pays consommateurs et/ou producteurs de semences, ont intérêt à mettre en place, comme le prévoient les accords de I'OMC, un système de protection des droits de l'obtenteur, afin que leur agriculture ne soit pas pénalisée et puisse bénéficier des avancées de la sélection. La mise en œuvre de ces droits devra se faire très progressivement, s'intéressant en priorité aux espèces correspondant à des semences à forte valeur ajoutée. C'est une nécessité immédiate, comme nous venons de le montrer, mais cela aura aussi l'avantage de pousser à une organisation du secteur des semences vivrières et à roder le système en vue de son application à ces dernières qui posent beaucoup plus de problèmes. 


\section{Problèmes spécifiques aux semences vivrières}

Alors que l'on assiste dans les pays développés depuis la fin de la Seconde Guerre mondiale à un accroissement rapide et continu de la productivité de la plupart des grandes spéculations agricoles (plus de 1 quintal/ha/an pour le blé en France sur les quarante dernières années), les rendements ont peu progressé, voire stagné, dans certains pays du Sud, principalement en Afrique, ne dépassant pas souvent 1 à 2 t/ha. Pourtant, ces pays ont bénéficié de l'aide internationale au travers de multiples plans de développement à tous les niveaux de la filière semences : sélection de nouvelles variétés, élaboration d'une législation semencière, création de centres semenciers, mise en place de réseaux d'essais ou d'échange de semences, soutien au secteur informel, etc. II n'y a pas dans toute I'histoire du développement agricole de sujet qui ait été autant débattu, fait couler autant d'encre et donné lieu à plus de projets ! Malheureusement, les résultats sont là : les petits agriculteurs de Madagascar, du Mali ou d'autres pays essentiellement africains continuent majoritairement à multiplier leurs propres semences, que ce soit par obligation (ils savent qu'elles ne sont pas les plus adaptées mais ils n'ont pas accès, pour des raisons techniques, financières ou autres, à des produits plus performants) ou par conviction (les variétés locales sont sensées fournir les meilleurs résultats). De plus, ils connaissent d'énormes difficultés de réapprovisionnement en cas de mauvaise récolte, accident de conservation, trouble politique, etc., alors que des bâtiments de stockage sont pratiquement vides et que des chaînes de conditionnement de semences surdimensionnées tournent en sous-régime. Essayons d'analyser les raisons de ces multiples échecs.

\section{Une approche quelque peu transcendantale des semences et des variétés}

Toute production végétale commence par la mise en terre d'un plant ou d'une semence, qui est sensé contenir tous les attributs de la variété (programme génétique) et porte en lui tout le potentiel de la future récolte. C'est donc à la fois un symbole et un héritage des anciens, mais aussi un bien matériel précieux, car il est le résultat du travail de la saison précédente et qu'il a fallu, pour en disposer au moment du semis, prendre tout un ensemble de précautions, et parfois même se priver ! Dans de nombreuses traditions, les semences ont une telle valeur qu'elles ne sauraient faire l'objet d'une transaction commerciale alors qu'elles peuvent être données ou échangées, constituer des gages d'amitié ou d'alliance, etc.

Une autre croyance est celle de la micro-adaptation variétale aux conditions locales, qui relève plus de l'idéologie que de l'analyse objective de résultats sur le terrain. L'expérience montre que, par définition, une bonne variété présente une bonne souplesse d'adaptation aux diverses contraintes biotiques et abiotiques - une bonne homéostase dans le jargon des généticiens - et que, par voie de conséquence, elle convient à une large zone agro-écologique. Tout est une question d'échelle. Qui plus est, ce concept de micro-adaptation variétale à des contraintes locales, qui conduit à une impasse sur le plan technique, est aussi complètement irréaliste du point de vue économique !

II reste que ces approches quelque peu idéalistes des semences constituent aujourd'hui encore, dans certaines régions du monde, un frein à la diffusion de nouvelles variétés. 


\section{Des contraintes politico-culturelles}

Localement, certaines plantes vivrières comme le blé, le riz, le maïs, la vigne, etc., correspondent à des traditions extrêmement fortes et il est politiquement incontournable, lorsque l'on s'intéresse aux semences dans un pays donné, que la priorité aille d'emblée à ces productions. Cela est le plus souvent justifié mais peut aussi conduire, dans certains cas, à des erreurs stratégiques ! En effet, ces productions traditionnelles sont, par définition, plutôt bien maîtrisées localement en termes de maintenance variétale et de production de semences et, tant que l'environnement sociologique, technique, économique, etc., n'évolue pas significativement, l'impact des efforts consacrés à l'amélioration des semences de ces productions ne peut être qu'extrêmement réduit. En revanche, d'autres productions végétales, justement parce qu'elles ne sont pas localement traditionnelles ou qu'elles bénéficient d'un environnement évolutif, peuvent réagir très rapidement et positivement à des programmes semenciers et variétaux bien ajustés. À titre d'exemple, on citera le cas de Madagascar où l'essentiel des efforts en matière de semences a toujours porté sur le riz (consommation avoisinant $200 \mathrm{~kg} /$ tête/an) alors que cette production continue, aujourd'hui, d'être déficitaire avec un rendement moyen en paddy stagnant depuis plus de 20 ans autour de 2 tonnes/ha. Il est raisonnable de penser que, si une partie des moyens mis sur le secteur rizicole avait été investie sur les productions maraîchères (ou fruitières), l'impact aurait été beaucoup plus significatif sur l'ensemble de l'économie malgache.

\section{La production semencière : une industrie lourde?}

La plupart des espèces vivrières se caractérisent par des taux de multiplication plutôt faibles, de l'ordre de 10 ou 15 pour l'arachide, à 300 pour le sorgho. Compte tenu des surfaces en jeu, les projets de développement ont eu à faire face d'emblée à des besoins représentant des volumes considérables. De plus, les doctrines économiques de l'après-guerre ayant été marquées par les avantages de la taylorisation et des économies d'échelle, les solutions ont été le plus souvent recherchées au travers de la mise en place de structures importantes, capables théoriquement de produire et distribuer des semences au niveau d'une grande région, voire d'un pays tout entier. Cependant, la plupart de ces centres semenciers qui disposaient chacun de surfaces importantes (parfois plusieurs centaines d'hectares, voire plus) n'ont pas répondu aux espoirs qui étaient fondés sur eux, et cela pour de multiples raisons : lourdeur de leur gestion, prix de revient élevé et qualité technique (identité variétale, germination, état sanitaire, etc.) médiocre des produits, faible réactivité à l'évolution de la demande variétale, coûts de transports des semences, etc.

\section{Une trop grande place de l'État}

Du fait que l'agriculture, et tout particulièrement les productions prises en charge par ces établissements semenciers, constituaient la base de l'économie du pays, il était naturel que les gouvernements en place, quelles que soient leurs options politiques, aient souhaité maîtriser ces projets qui représentaient pour eux un enjeu économique d'importance stratégique. De plus, les bailleurs de fonds ont pendant longtemps manifesté " une certaine retenue " pour apporter leur concours à des projets de statut strictement privé ou dans lesquels un État n'était pas représenté avec un poids significatif, ceci avec le légitime souci de disposer de meilleures garanties quant à la gestion et à l'utilisation des fonds mis à disposition. 
Ainsi, que ce soit dans le cadre de sociétés d'État ou de sociétés d'économie mixte, les activités de production et de commercialisation de semences ont souvent été éloignées du monde des fermiers et autres petits producteurs.

\section{Des formes d'aides parfois inadéquates}

Nombre de petits agriculteurs des pays les moins avancés ont de grandes difficultés pour disposer des semences qui leur sont nécessaires au moment des semis (manque de trésorerie, conflit, sécheresse, etc.). Divers systèmes ont été imaginés pour pallier ces difficultés mais aussi pour faire la promotion des semences dites " sélectionnées " ou " commerciales ", issues des centres ou établissements semenciers évoqués ci-dessus. Mais parfois avec des effets non souhaités.

C'est tout d'abord la " banque de semences ", surtout employée pour les espèces autogames à très faible taux de multiplication comme l'arachide. Dans la pratique, on constate rapidement une dérive dans la qualité des semences retournées (pureté variétale, état sanitaire, germination, etc.) entraînant une dégradation de l'efficacité du système et un désintérêt progressif des agriculteurs.

Une autre voie a été de vendre à bas prix, voire de distribuer gratuitement les semences sélectionnées. Ces opérations ont eu, dans quelques cas, deux sortes d'effets pervers : d'une part, la dévalorisation du produit aux yeux des agriculteurs qui en bénéficiaient, certains d'entre eux allant même jusqu'à refuser d'envisager l'achat de semences à l'avenir ${ }^{4}$; d'autre part, du fait de leur prix attractif, particulièrement en période de soudure, le détournement de ces semences sélectionnées directement vers la consommation!

\section{Déconnexion des semences avec les autres facteurs de production}

On oublie trop souvent que la variété (programme génétique) et la semence (son support physique) ne sont que deux facteurs de production parmi d'autres : qualité des sols, ressources en eau, alimentation minérale, protection contre le parasitisme ou l'enherbement, etc. Proposer des semences à haut potentiel sans pouvoir leur assurer les conditions qui leur permettront de s'exprimer revient à poser un emplâtre sur une jambe de bois. Lorsque les rendements d'un riz, d'un sorgho ou d'un maïs ne dépassent pas 20 quintaux/ha, qu'une arachide ou un soja n'atteint pas les $400 \mathrm{~kg} / \mathrm{ha}$, la semence peut être en cause en cas de mauvaise germination, mais ce n'est jamais un problème dû à la variété en soi !

Grâce à l'expérience acquise dans les pays développés, on peut envisager de brûler les étapes du développement sous les climats plus contraignants, encore faut-il se donner les moyens d'une évolution harmonieuse en veillant à une progression équilibrée de l'ensemble des facteurs de production.

\section{Des objectifs de sélection ou des préconisations variétales inadaptées}

Dans cet examen des raisons des succès mitigés, voire des échecs de projets semenciers, il faut également signaler des choix inopportuns qui ont pu être faits en amont ou en aval de la production des semences, en matière d'orientation de la sélection ou au niveau du conseil variétal sur le terrain. 
Un exemple classique, à défaut de pouvoir en citer un autre, est celui de deux options d'un important programme d'amélioration génétique du sorgho, céréale par excellence des zones à ressources en eau limitées, développé dans les années 60-70 en Afrique du Centre et de l'Ouest : d'une part, la création de variétés à fort potentiel pour les situations non limitantes (zones de bas-fonds ou avec irrigation) et, d'autre part, la mise au point de variétés à cycle court, afin d'exploiter au mieux une saison des pluies de plus en plus réduite. L'utilisation de géniteurs américains permettant de s'abstraire de la photopériodicité des variétés traditionnelles s'est traduite par des caractères défavorables au niveau des grains des variétés issues du premier cycle de sélection : une faible teneur en amylose (c'est-à-dire à tendance farineuse, aboutissant à un tô, base de l'alimentation locale, moins apprécié de par sa consistance et sa moins bonne conservation) ainsi qu'une moins bonne tolérance aux insectes durant le stockage (pertes plus élevées). En fait, il s'agissait d'un lancement trop hâtif de ces variétés qui auraient pu être corrigées par un second cycle de sélection.

Par ailleurs, dans le cadre de programmes de développement plutôt dirigistes, des choix variétaux, non toujours bien inspirés, ont parfois été imposés aux agriculteurs de toute une région. Un exemple est le cas du riz hybride en Chine, où le gouvernement avait fait de son développement un enjeu national, démarré vers 1975. Au travers d'une croissance régulière, les surfaces en riz hybrides ont dépassé 17 millions d'hectares au début de 1991, représentant alors plus de $52 \%$ de la surface totale (33 millions d'hectares). Cependant, la qualité technologique des grains (en particulier un taux de brisure très élevé) de ces variétés hybrides était peu prisée des consommateurs chinois et, lorsque la politique " volontariste " du gouvernement chinois fit place à partir de 1992 à une plus grande liberté de choix variétal, les surfaces en riz hybrides diminuèrent rapidement pour se stabiliser aujourd'hui (dans l'attente de variétés améliorées sur le plan technologique) autour de 13 millions d'hectares (environ $40 \%$ ).

\section{L'absence de filières produits}

Un dernier aspect qui handicape le développement des semences de produits vivriers est le fait que, comme leur nom l'indique, leur production est en très grande partie autoconsommée et que le reste est commercialisé par des circuits cours (marchés locaux). De ce fait, il n'y a pas le plus souvent de demande organisée pour des types ou des qualités de grain, de farine, de cossette, etc., ni de paiement à la qualité. Ces aspects étant très largement sous déterminisme génétique, il n'y donc pas d'incitation à épurer les semences que l'on resème ou à renouveler la variété.

Pourtant, l'expérience montre toujours que les agriculteurs sont extrêmement réactifs, positivement et négativement, à la notion de qualité pour peu qu'elle soit associée à une politique de prix correcte, même si les quantités qui vont sur le marché sont minimes. Avec en plus une répercussion favorable sur les productions strictement autoconsommées, l'agriculteur leur appliquant les mêmes techniques. 


\section{Une solution : la production de semences sous label}

II ressort clairement de ce qui précède que l'urgence en matière de semences vivrières dans les pays du Sud ne se situe pas au niveau de la protection de la propriété intellectuelle des variétés. Dans un premier temps, la priorité doit aller à la mise en place de filières semences, utilisant et valorisant les ressources et savoir-faire locaux, afin d'assurer leur autonomie et leur viabilité financières pour l'immédiat et leur capacité à évoluer pour le long terme.

\section{Variétés traditionnelles/variétés modernes}

Compte tenu des variétés traditionnelles existantes et des efforts de sélection qui ont été conduits pendant tout le siècle dernier - par les centres internationaux de recherche agronomiques (IRRI, CIAT, Cimmyt, CIP, etc.), divers organismes de coopération internationale français (Cirad, IRD) ou étrangers, etc. - les pays du Sud disposent d'emblée, pour la plupart de leurs grandes productions vivrières, d'un accès à une très large palette de matériel génétique valable qu'il convient d'exploiter en priorité. Ces variétés ont pour la plupart été déjà évaluées, dans de multiples situations, durant la même période et il serait vraiment incohérent de recommencer ce travail colossal.

Il s'agit donc dans un premier temps d'exploiter tous les résultats existants, de façon à ne reprendre qu'un nombre minimum de ces cultivars dans des essais de confirmation qui devront être permanents car chaque année amène de nouveaux candidats. Très rapidement sera ainsi mise en évidence une gamme de produits adaptés aux conditions et aux besoins de chaque grande région. Cette activité devrait relever, dans l'attente d'un relais par le secteur privé, des instituts, stations ou centres nationaux de recherche agronomique.

\section{Catalogue officiel}

À partir de ces résultats, un catalogue officiel des variétés cultivées doit être établi et géré au niveau national $\left.\right|^{5}$ par un comité où sont représentés tous les partenaires concernés : État, recherche, sélectionneurs, professionnels de la semence, agriculteurs, industriels, consommateurs, etc. L'intérêt de ce catalogue est double : d'une part, il constitue une référence pour les agriculteurs en leur indiquant les variétés les plus performantes ou les plus adaptées; d'autre part, il contient les fiches descriptives de chacune des variétés, ce qui permet de les identifier et d'évaluer leur conformité génétique tout au long du processus de maintenance et de multiplication des semences.

\section{Le rôle essentiel de la maintenance variétale et la production des semences de pré-base}

Quel que soit le type variétal (lignée, population, clone multiplié végétativement, etc.), la pureté et l'état sanitaire des variétés se dégradent au fil des générations de multiplication si des procédures strictes ne sont pas appliquées. C'est le constat fataliste, entendu partout : « les variétés dégénèrent $» !$

Pourtant, la maintenance rigoureuse des variétés ne demande ni moyens financiers importants, ni équipements coûteux mais exige des parcelles homogènes, du temps, des dispositifs et des savoirfaire spécifiques... et beaucoup de rigueur ! Pour chaque variété, elle met en jeu des quantités modestes (de l'ordre de quelques kilos ou dizaines de kilos) et peut être réalisée par le secteur public ou par un opérateur privé qui en prend la responsabilité. 
Ne serait-ce que pour mieux asseoir leur rémunération, ces mêmes mainteneurs auront intérêt à étendre leur activité à la production des premières générations (semences de pré-base et base) qui mettent en jeu des quantités ne dépassant pas quelques tonnes.

\section{Une production de semences confiée (principalement) au secteur privé}

À partir des semences de base fournies régulièrement par les mainteneurs, la production des semences commerciales de plantes vivrières doit être assurée par des agriculteurs-multiplicateurs de semences (AMS) soit intervenant à titre personnel, soit regroupés au sein de groupements ou d'établissements semenciers. Au départ, on favorisera le démarrage d'un grand nombre d'initiatives locales de façon à assurer un maillage du terrain permettant un contact étroit avec les agriculteursproducteurs de produits de consommation (APC), à fournir les semences au moindre coût (pas de transport), à suivre et à réagir à l'évolution de la demande.

Les AMS seront bien sûr des volontaires, mais dont la candidature sera examinée sur la base d'un certain nombre de critères qui devront être, entre autres : la technicité reconnue, la participation régulière à des formations sur la production de semences, l'adhésion à un guide de bonnes pratiques semencières, un équipement minimum en matière de conditionnement et de stockage des semences (tamis, mini silos individualisés, etc.), plus l'engagement de n'utiliser comme semences mères des semences commerciales que des semences de base dûment certifiées (assurant la rémunération des mainteneurs).

Il appartiendra à un service de l'État, généralement désigné sous le nom de Service officiel de contrôle ou SOC (bien que, dans le schéma d'organisation proposé ici, cette dénomination soit peu adaptée), de délivrer un " droit à produire " ou une "licence de production " des semences commerciales, pour des variétés et des volumes définis, impliquant l'achat d'une quantité définie de semences de base et la remise d'un nombre correspondant de certificats ${ }^{6}$ qui devront obligatoirement accompagner les semences commerciales.

\section{Les rôles du Service officiel de contrôle}

Dans ce schéma, l'État conserve toutes ses prérogatives mais il devra les exercer dans un esprit plus de conseil et de formation que de contrainte et de sanction. En particulier, il lui appartient, dans le cadre d'une concertation avec tous les partenaires de chaque filière produit, de fixer les normes techniques relatives à chaque génération de multiplication des semences.

Par son service de contrôle, il interviendra classiquement auprès des mainteneurs et des producteurs de semences de base, afin que le matériel de multiplication fourni aux AMS soit conforme à ces normes. La charge de ce service sera de ce point de vue considérablement allégée, les premières générations de multiplication étant concentrées chez les mainteneurs, qui seront le plus généralement des stations de recherche puis, les activités se développant, des établissements semenciers privés. 
Ainsi déchargé, le SOC pourra se consacrer à son rôle prioritaire qui est d'assurer le soutien technique des AMS par des stages, des visites sur le terrain, des conseils, etc. Il exercera cependant à l'égard de ces derniers, par des sondages systématiques, le suivi des bonnes pratiques semencières et de la qualité des semences mises sur le marché par rapport aux normes en vigueur. En cas de défaillance constatée, des sanctions, pouvant le cas échéant bénéficier d'une certaine publicité, seront appliquées : avertissement, réduction du droit à produire, retrait de la licence.

Ces mesures, simples d'application, devraient se révéler efficaces car elles présentent un double avantage : d'une part, en responsabilisant les AMS devant leurs futurs clients, elles assurent une bonne garantie de qualité des semences commerciales; d'autre part, elles vont permettre aux AMS les plus dynamiques et compétents de développer leur activité, voire de créer leur propre société semencière.

\section{Notes:}

${ }^{1}$ Accord sur les aspects des droits de propriété intellectuelle qui touchent au commerce. En anglais TRIPS (Trade Related aspects of Intellectual Property rightS).

${ }^{2}$ Rappelons qu'au 6 septembre 2001, la Convention Upov comptait 49 pays adhérents et qu'environ 120 pays ont adopté ou sont en cours d'adoption d'une législation semencière conforme aux principes de I'Upov. En particulier, dans le cadre de I'OAPI (Office africain de la propriété intellectuelle), 16 pays d'Afrique de l'Ouest et du Centre ont adopté début 2000, à l'occasion de la révision de l'accord de Bangui de 1977, un système complet de protection de la propriété intellectuelle, y compris un volet "variété végétales » conforme à l'Upov. Cet accord devrait entrer en vigueur à partir de sa ratification par au moins 10 des pays signataires.

${ }^{3}$ C'est un point qui a été complètement obéré par les détracteurs du système, injustement qualifié de " terminator ", qui visait à pouvoir créer des variétés hybrides de plantes autogames. Le premier résultat concret de cette campagne de dénigrement aura été de détourner nombre d'entreprises privées de tout programme de sélection de plantes autogames, tout particulièrement pour les régions du monde n'ayant pas mis en place une protection des droits de l'obtenteur.

${ }^{4}$ Réponses à une enquête de motivation pour l'achat de semences sélectionnées par les agriculteurs de plusieurs régions de Madagascar, conduite en 1999 dans le cadre d'une étude financée par l'Agence française de développement.

${ }^{5}$ Dans un deuxième temps, ces catalogues pourraient être de nature supranationale, afin d'en partager la charge, si le contexte agro-écologique le justifie.

${ }^{6}$ II peut s'agir tout simplement d'étiquettes (éventuellement numérotées) portant le nom du producteur, le nom de la variété, le $n^{\circ}$ du lot, etc. 


\section{CONCLUSION}

\section{...des variétés traditionnelles aux variétés protégées}

Il s'agit donc de mettre en place une production de "semences sous label " en substituant, afin d'alléger le système lourd, coûteux et parfois arbitraire de la certification des semences commerciales, l'obligation de résultats par une obligation de moyens. Cette organisation doit se développer aux conditions économiques locales, ces " semences sous label " devant être offertes aux coûts qui sont déjà pratiqués pour ce type de produit. Elle devra s'appliquer dans un premier temps à toutes les variétés déjà disponibles (relevant du domaine public), traditionnelles ou issues de sélection, qui sont à même de répondre aux besoins immédiats d'une grande majorité des agriculteurs du Sud. Elle offre même le cadre idéal pour relancer ces produits, les faire connaître ou reconnaître, leur redonnant toute leur valeur et leur utilité.

Ce faisant, cette organisation préparera la voie aux variétés modernes susceptibles d'être protégées par l'Upov ou un système sui generis équivalent, en offrant deux conditions nécessaires à leur développement : la garantie, grâce au label, de pouvoir proposer aux agriculteurs une semence de qualité, conforme au prototype mis au point par le sélectionneur ; une organisation viable économiquement et capable d'évoluer, par la possibilité d'un retour sur investissement (au travers de royalties perçues sur les semences de base et éventuellement sur les semences commerciales dont les quantités sont contrôlées).

Le contexte ainsi créé devrait permettre de redynamiser tout le secteur de la sélection, que ce soit par le redéploiement ou la création d'entreprises publiques ou privées au niveau local, ou par la mobilisation d'entreprises internationales qui trouveront là l'opportunité de nouveaux marchés.

\section{Illustrations}

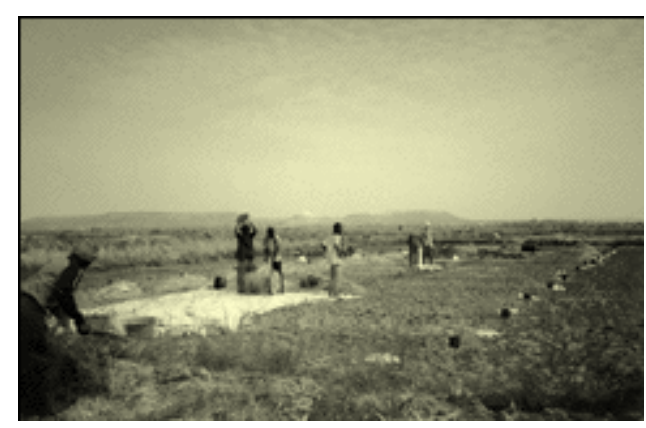

Photo 1. Dispositif pour la maintenance d'une variété de riz en Mauritanie : battage individuel des familles sélectionnées. 


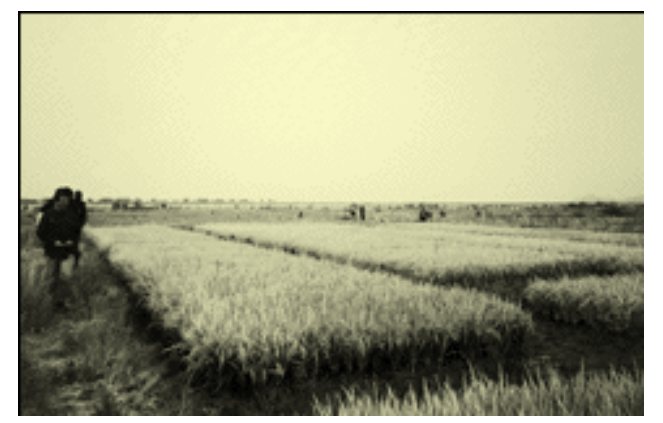

Photo 2. Dispositif pour la multiplication des premières générations (pré-bases) d'une variété de riz en Mauritanie. 